- d'évaluer les menaces à partir de la recherche, du recueil et de l'exploitation du renseignement et de définir les menaces de référence à partir de ces données et de les notifier aux opérateurs ;

- de tenir les opérateurs informés des menaces qui pèsent sur leurs installations ou activités ;

- de disposer d'une capacité de réponse et d'intervention suffisante grâce aux forces de l'ordre disponibles aux niveaux local, régional et national ;

- de définir les règles nécessaires en termes de confidentialité et de protection des informations sensibles.

Les opérateurs sont, quant à eux, notamment tenus de mettre en place des dispositions :

- de détection des actions malveillantes ainsi que des intrusions ou tentatives d'intrusion ;

- d'alerte et d'information des pouvoirs publics et, le cas échéant, des organismes impliqués (par exemple le centre opérationnel dédié de l'IRSN en cas d'événement affectant un transport de matières nucléaires) ; l'alerte doit être accompagnée d'une première évaluation de la situation ;

- de retardement de nature à entraver ou gêner l'action des agresseurs d'une installation ou d'un transport.

Les dispositions à mettre en œuvre pour limiter les conséquences d'un acte de malveillance sont quant à elles définies en étroite collaboration entre les opérateurs et les pouvoirs publics, notamment dans le cadre des plans d'urgence élaborés par ces différentes entités et traitant respectivement des aspects sûreté et sécurité.

\title{
1.4. Instances gouvernementales concernées
}

$\mathrm{Au}$ sein des pouvoirs publics, plusieurs entités sont impliquées dans le dispositif de sécurité nucléaire. Elles exercent, en étroite collaboration, des responsabilités complémentaires.

\subsubsection{Secrétariat général de la défense et de la sécurité nationale}

Le Secrétariat général de la défense et de la sécurité nationale (SGDSN) est un service du Premier ministre. Il a un rôle général de coordination interministérielle en matière de politique de défense et de sécurité et se trouve notamment chargé de la planification de la gestion de crise dans le domaine nucléaire. À ce titre, le SGDSN est en charge de l'élaboration et des modifications de la réglementation relative aux activités d'importance vitale, qui comprend la définition des menaces à prendre en compte. Le SGDSN est en outre chargé de la définition de la politique de confidentialité et de l'élaboration des règles de mise en œuvre associées. 


\subsubsection{Ministre chargé de l'Énergie}

La responsabilité du contrôle des matières nucléaires est confiée par le code de la défense au ministre chargé de l'Énergie pour les matières nucléaires à usage civil. À ce titre, ce ministre est en charge :

- de l'élaboration et des modifications de la réglementation applicable en ce domaine aux matières et installations nucléaires et aux transports ;

- de la délivrance des autorisations nécessaires aux opérateurs pour exercer une activité impliquant des matières nucléaires et des mises à jour de ces autorisations ;

- de l'exercice du contrôle de la mise en œuvre par les opérateurs des dispositions prescrites dans les autorisations qui leur ont été délivrées ; il diligente notamment des inspections sur les sites nucléaires et au cours de transports ;

- de l'application, dans le secteur nucléaire, de la réglementation relative aux activités d'importance vitale ;

- de l'organisation d'exercices de crise nationaux.

Pour exercer ces responsabilités, le ministre chargé de l'Énergie s'appuie sur un service dédié constitué de personnels en charge de l'instruction des dossiers, de l'inspection et de l'élaboration de la réglementation. Ce service est placé sous la responsabilité du Haut fonctionnaire de défense et de sécurité auprès du ministre en charge de l'Énergie (HFDS) ; le HFDS assure la fonction d'autorité compétente pour la sécurité nucléaire. Pour l'exercice de ses missions, le HFDS fait appel, en tant qu'appui technique à l'IRSN, établissement public à caractère industriel et commercial (§ 1.4.6).

\subsubsection{Ministre de l'Intérieur}

Le ministre de l'Intérieur a autorité sur l'ensemble des forces de l'ordre, tant locales que nationales, susceptibles d'intervenir en cas d'acte de malveillance. Selon la localisation des installations nucléaires ou des transports, les forces de l'ordre compétentes appartiennent à la gendarmerie nationale ou à la police nationale. Dans tous les cas, des protocoles régissent les relations entre ces forces de réponse dépendant des pouvoirs publics et celles mises en place par les opérateurs. De plus, les services de renseignement qui dépendent du ministre de l'Intérieur ont un rôle essentiel dans la prévention des actes de malveillance et contribuent à l'évaluation des menaces.

Par ailleurs, sur décision du Premier ministre, la conduite interministérielle de la réponse opérationnelle à une crise peut être confiée à ce ministre.

\subsubsection{Préfet de département}

Dans chaque département, un préfet dirige l'action de l'État. À ce titre, il est notamment responsable de la gestion locale de toutes les crises se produisant dans son département, et en particulier de celles, d'origine accidentelle ou résultant d'un acte 
de malveillance, pouvant affecter une installation nucléaire ou un transport de matière radioactives. Ce rôle essentiel du préfet de département en cas de crise a conduit à lui confier l'approbation du plan particulier de protection (PPP) — préparé par chaque opérateur - et la réalisation du plan de protection externe (PPE), tels que prévus par la réglementation relative aux activités d'importance vitale (§ 1.6.1).

\subsubsection{Autorité de sûreté nucléaire (ASN)}

Dans le cadre de sa mission de contrôle, l'ASN analyse les risques et inconvénients que les installations nucléaires et les transports de matières radioactives peuvent présenter pour la sécurité, la santé et la salubrité publiques ou pour la protection de la nature et de l'environnement, quelle que soit l'origine de ces risques (malveillance ou non). Le cas échéant, l'ASN notifie aux opérateurs les prescriptions nécessaires à la protection des personnes du public et de l'environnement.

L'ASN est consultée sur les projets de décrets et d'arrêtés ministériels relatifs à la sécurité nucléaire.

En cas de situation d'urgence radiologique, quelle qu'en soit l'origine, l'ASN assure une mission de conseil auprès des pouvoirs publics (notamment le préfet, le ministre en charge de la gestion de la crise et le Premier ministre) dans le domaine de la protection des populations et de l'environnement. L'ASN a également pour mission de s'assurer que l'opérateur prend les bonnes dispositions pour ramener à l'état sûr l'installation concernée par une action de malveillance.

\subsubsection{Institut de radioprotection et de sûreté nucléaire}

L'IRSN, EPIC créé en $2002^{4}$, apporte un soutien technique à l'ensemble des autorités gouvernementales impliquées dans la sécurité des matières nucléaires, de leurs installations et de leurs transports. En particulier, l'IRSN effectue, pour le compte du HFDS auprès du ministre chargé de l'Énergie et dans le cadre d'une convention passée avec ce ministère, les missions suivantes :

- il réalise des analyses de situations ou de dossiers en vue de préparer des décisions du HFDS ;

- il met à disposition du HFDS des personnels habilités et assermentés en vue d'effectuer des inspections dans les installations nucléaires et sur les transports de matières nucléaires ;

- il participe à l'élaboration de la réglementation et assiste les services du HFDS dans les activités menées à cet égard à l'international ;

- il tient une comptabilité centralisée de l'ensemble des matières nucléaires civiles détenues en France ;

4. L'IRSN résulte de la fusion de l'IPSN (Institut de protection et de sûreté nucléaire), qui faisait partie du CEA et de l'OPRI dans sa composante technique (Office de protection contre les rayonnements ionisants). 
- il assure la gestion et le traitement des accords d'exécution des transports de matières nucléaires, le suivi opérationnel de ces transports et la transmission des alertes aux autorités ;

- il mène des études ou des expérimentations en support à ses expertises techniques ou pour les besoins des contrôles réalisés lors des inspections.

\subsection{Cadre réglementaire}

La réglementation française applicable aux matières nucléaires, à leurs installations et à leurs transports comporte des textes de niveau législatif (lois), et des textes de niveau réglementaire (décrets et arrêtés d'application). Ces textes se trouvent principalement dans le code de la défense ou en découlent ; il s'agit pour l'essentiel :

- des parties législative (articles L. 1333-1 et suivants du code de la défense) et réglementaire (articles R. 1333-1 et suivants) relatives à la protection et au contrôle des matières nucléaires ;

- des parties législative (articles L. 1332-1 et suivants du code de la défense) et réglementaire (articles $\mathrm{R}$. 1332-1 et suivants) relatives à la protection des installations d'importance vitale (§ 1.6.1);

- de la directive nationale de sécurité (DNS) du secteur nucléaire prise en application du code de la défense (document classifié non public précisant les menaces de référence) ;

- des arrêtés d'application des articles L. 1333-1 et suivants du code de la défense :

- arrêté relatif aux modalités d'une demande d'autorisation et à la forme de cette autorisation (application de l'article L. 1333-2 du code de la défense) ;

- arrêté relatif à la protection physique des installations abritant des matières nucléaires dont la détention suppose une autorisation ;

- arrêté relatif aux modalités de réalisation de l'étude prévue à l'article R. 1333-4 du code de la défense pour la protection des matières nucléaires et de leurs installations ;

- arrêté fixant les conditions de mise en œuvre du suivi physique et de la comptabilité des matières nucléaires dont la détention suppose une autorisation ;

- arrêté relatif à la protection et au contrôle des matières nucléaires en cours de transport ;

- arrêté relatif aux mesures de suivi physique, de comptabilité et de protection physique applicables aux matières nucléaires faisant l'objet d'une déclaration ainsi qu'à la forme et aux modalités de cette déclaration ;

- arrêté du ministre chargé de l'Énergie relatif à la composition et au fonctionnement des groupes d'experts sur la sécurité des installations, ouvrages et transports nucléaires. 